\title{
Design and Implementation of FPU for Optimised Speed
}

\author{
R. Bhuvanapriya, Menakadevi T.
}

\begin{abstract}
Currently, each CPU has one or additional Floating Point Units (FPUs) integrated inside it. It is usually utilized in math wide-ranging applications, such as digital signal processing. It is found in places be established in engineering, medical and military fields in adding along to in different fields requiring audio, image or video handling. A high-speed and energy-efficient floating point unit is naturally needed in the electronics diligence as an arithmetic unit in microprocessors. The most operations accounting $95 \%$ of conformist FPU are multiplication and addition. Many applications need the speedy execution of arithmetic operations. In the existing system, the FPM(Floating Point Multiplication) and FPA(Floating Point Addition) have more delay and fewer speed and fewer throughput. The demand for high speed and throughput intended to design the multiplier and adder blocks within the FPM (Floating point multiplication)and FPA(Floating Point Addition) in a format of single precision floating point and double-precision floating point operation is internally pipelined to achieve high throughput and these are supported by the IEEE 754 standard floating point representations. This is designed with the Verilog code using Xilinx ISE 14.5 software tool is employed to code and verify the ensuing waveforms of the designed code.
\end{abstract}

Keywords: FPU, FPM, FPA, IEEE 754,Xilinx ISE.

\section{INTRODUCTION}

Floating point units (FPUs) are a mathematical coprocessor that's just for floating point numbers. It will handle arithmetic operations. The most newest processors through software library routines can also accomplish numerous transcendental functions like exponential or trigonometric calculation. In computer the real numbers can be expressed in numerous ways. Floating-point representation mainly follows the standard IEEE-754 format, which is the foremost common way of representing an approximate to real numbers in computers as it's well handled in most bulky processors. The real numbers represented in binary format are called floating point numbers. These are portrayed computers by a standards Committee which was formed in 1985 by IEEE .The IEEE-754 floating point illustration for binary real numbers contains three parts is shown in figure 1.

\begin{tabular}{|l|l|l|} 
SIGN & EXPONENT & MANTISSA \\
\hline
\end{tabular}

\section{Fig.1: Floating point representation}

Revised Manuscript Received on February 05, 2020.

* Correspondence Author

R. Bhuvanapriya*, P.G. Student, Department of ECE, Adhiyamaan College of Engineering, Hosur, Tamil nadu, India.

E-mail: bhuvanapriya.ramkumar@gmail.com

Dr. Menakadevi T., Associate Professor, Department of ECE, Adhiyamaan College of Engineering, Hosur, Tamil nadu, India.

E-mail: menaka_sar@rediffmail.com

(c) The Authors. Published by Blue Eyes Intelligence Engineering and Sciences Publication (BEIESP). This is an open access article under the CC BY-NC-ND license (http://creativecommons.org/licenses/by-nc-nd/4.0/)

\section{A. Floating Point Representation}

The floating point are often represented in four main sizes such as half (16 bits) ,single(32 bits),double(64 bits) and quadruple (128 bits) precision. The IEEE single precision floating point format has a sign bit, 8 bits of exponent, 24 bits of mantissa and occupies 32 bit. The value of bias is 127 , an exponent 0 implies that -127 is kept within the exponent field. The exponents -127 (all 0s) and +128 (all 1s). The mantissa/significand is 24 bits long -23 bits of the mantissa that is stored in the memory and an implied ' 1 ' as the most significant' $24^{\text {th }}$ 'bit.Thus, a (32 bit) single precision floating point number representation in the IEEE standard is given by equations(1)\&(2).

$$
\mathrm{X}=\left(-1^{\mathrm{S}}\right) \times 2^{(\text {E-Bias })} \times(1 . \mathrm{M})
$$

Value $=\left(-1^{\text {Sign bit }}\right) \times 2^{(\text {Exponent-127) }} \times(1$.Mantissa $)$

The detailed range of the bit ranges of single precision and double precision is represented in Table I. The IEEE double precision floating point format has a sign bit,11 bits of exponent,53 bits of mantissa and occupies 64 bits overall. The exponent uses a biased representation with a bias of 1023. Thus, 64 bit double precision floating point number representation in the IEEE standard is given by equations (3).

Value $=(-1$ Sign bit $) \times 2$ (Exponent-1023) $\times(1$. Mantissa $)$
\begin{tabular}{|l|c|c|c|c|c|c|}
\hline PRECISION & SIGN & EXPONENT & Emax & Emin & $\begin{array}{c}\text { MANTISS } \\
\text { A }\end{array}$ & BIAS \\
\hline $\begin{array}{l}\text { Single } \\
\text { Precision }\end{array}$ & $1[31]$ & $8[30-23]$ & +127 & -126 & $23[22-0]$ & 127 \\
\hline $\begin{array}{l}\text { Double } \\
\text { Precision }\end{array}$ & $1[63]$ & $11[62-52]$ & +1023 & -1022 & $52[51-0]$ & 1023 \\
\hline
\end{tabular}

Table I: Bit Range

\begin{tabular}{|l|c|c|}
\hline PRECISION & MAX & MIN \\
\hline Single Precision & $3.40^{*} 10^{38}$ & $1.8^{*} 10^{-38}$ \\
\hline Double Precision & $1.8 * 10^{308}$ & $2.23 * 10^{-308}$ \\
\hline
\end{tabular}

Table II :IEEE 754 Standard Ranges

The IEEE 754 Standard range of maximum and minimum values that the single (32 bit) and double (64 bit) precision that can accommodate is shown in Table II. If the values exceeds the maximum value then its overflow case and when the values is below the minimum range then its underflow case .

In FPM and FPA internal adders and multipliers constitute an essential element as it performs $95 \%$ of operations , so with extensive research is focused on improving its performance and delays. 
Basically arithmetic operations like addition and multiplications to be implemented in digital computers. Amongst every arithmetic operation once the implementation of addition completed then it is easy to achieve multiplication.

This paper is ordered like: Section II describes work related to the implementation ,the system architecture is given in Section III, Section IV presents single precision floating point, Section V presents 32 bit FPM and followed by section VI presents implementation of 32 bit FPM and section VII presents 32 bit FPA and section VIII presents implementation of 32 bit FPA and section IX presents double precision floating point, section $\mathrm{X}$ presents pipelining technique ,section XI implementation of pipelined 64 bit FPM and section XII presents the implementation of pipelined 64 bit FPA and section XIII presents simulation results and section XIV presents conclusion and XV refers to future scope .

\section{II.RELATED WORK}

Several researchers have worked on implementing floating point arithmetic in their own way. The idea of floating point numbers given and three fields includes sign, fraction and an exponent field and extremely small to huge numbers with a varying level of precision [1]. The design of an IEEE single precision floating point addition and multiplication unit format given by IEEE std.754-1985 is characterized [2]. The IEEE-754 Standard defining numerous floating point number format and the sizes of the fields [3].The design of double precision floating point multiplier was implemented on FPGA [4].The concept of double precision floating point addition and subtraction for arithmetic unit was designed using the IEEE 754 format [5].The concept of pipelining is referred [6].This paper states with comparison of other adders CLA is better [9].The anatomy of floating point number concept referred [10]. The concept of representation of floating point number for single and double is stated.[11]

\section{ARCHITECTURE OF THE SYSTEM}

The floating point unit is an IC which is intended to govern all the arithmetic operations based on floating point numbers or fractions. Figure 2, shows the architecture of proposed FPU, this unit shows that there are pre-normaliser block for add and multiplication units and post normaliser block for both add and multiplication and the later exceptions unit is present to handle the expectations unit.The fpu_op helps in choosing the operation based on the inputs the operation is performed and the op_a and op_b are the operands and these are the floating point numbers will perform the appropriate functions based on the fpu_op operation . The fundamental microprocessor isn't capable of manipulating floating number quickly so a separate specialized floating point unit is designed as co-processor. This unit is meant to perform basically five operations like addition, subtraction, multiplication , division and square root. The proposed FPU corresponds to two major units in the unit which is represented in the top view of FPU is shown in Figure 3.

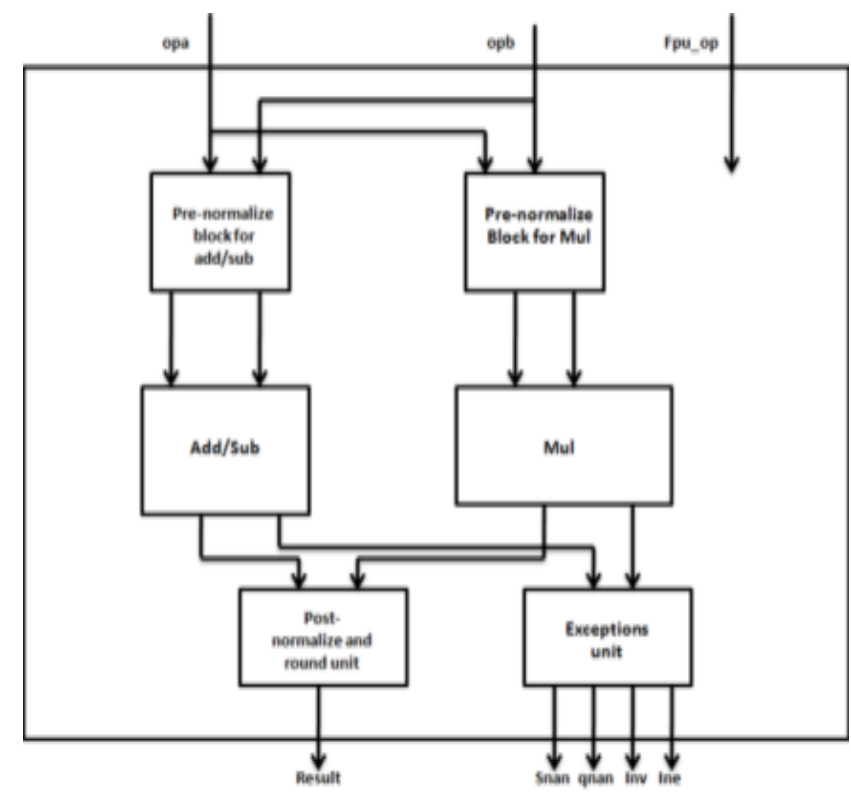

Fig 2: Architecture of proposed FPU

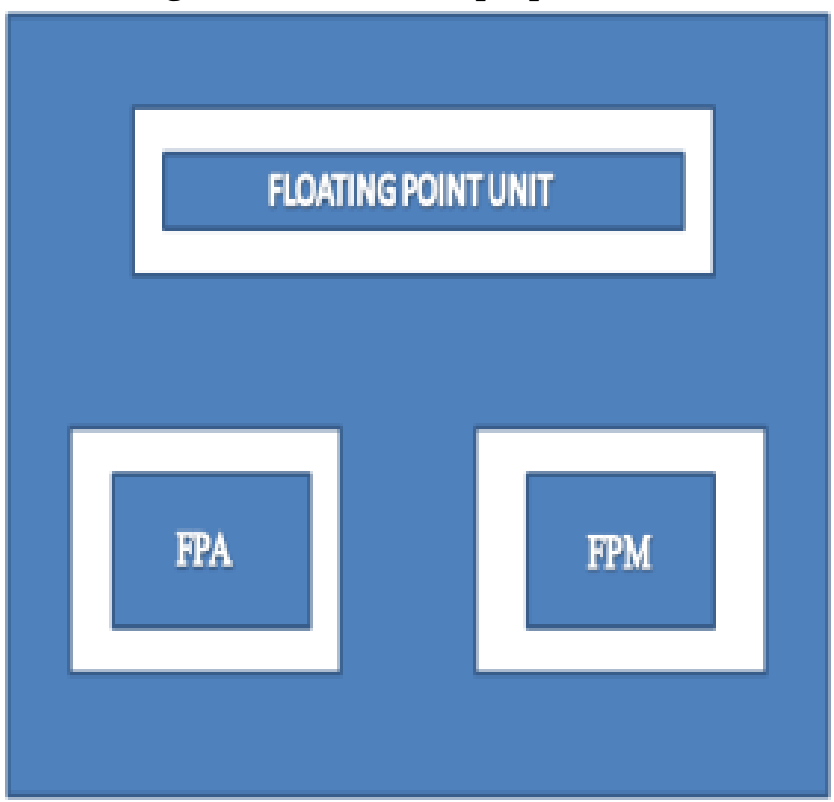

Fig 3: Top view of proposed FPU

\section{SINGLE PRECISION FLOATING POINT}

The IEEE single precision floating point format has a sign bit is represented as " $1 / 0$ " bit. " 1 " in sign bit indicates the negative and " 0 " indicates the positive. The total number of exponent is 255 here the ranges depends on the exponent value in accordance 8 bits of exponent is accommodated and it has a bias of 127 .There are totally 24 bits of mantissa but last 1 bit is hidden so it's always represented as 23 bit of mantissa as per IEEE-754 standard and occupies 32 bit.

\section{V.FLOATING POINT MULTIPLICATION}

Floating point multiplication (FPM) are represented by single precision format involve in estimation of sign bit , exponent and mantissa of the product and later normalizing and round off the resultant values to finally attain the result .The two operands are intended for XOR operation for the sign check . 
The exponents $\mathrm{A}$ and $\mathrm{B}$ are added to attain the resultant exponent is subtracted from 127 to achieve its biased exponent. The mantissa are multiplied based on the resultant results obtained .Finally, all are formed into the format declared by IEEE 754.The architecture of FPM is shown in figure 4 .

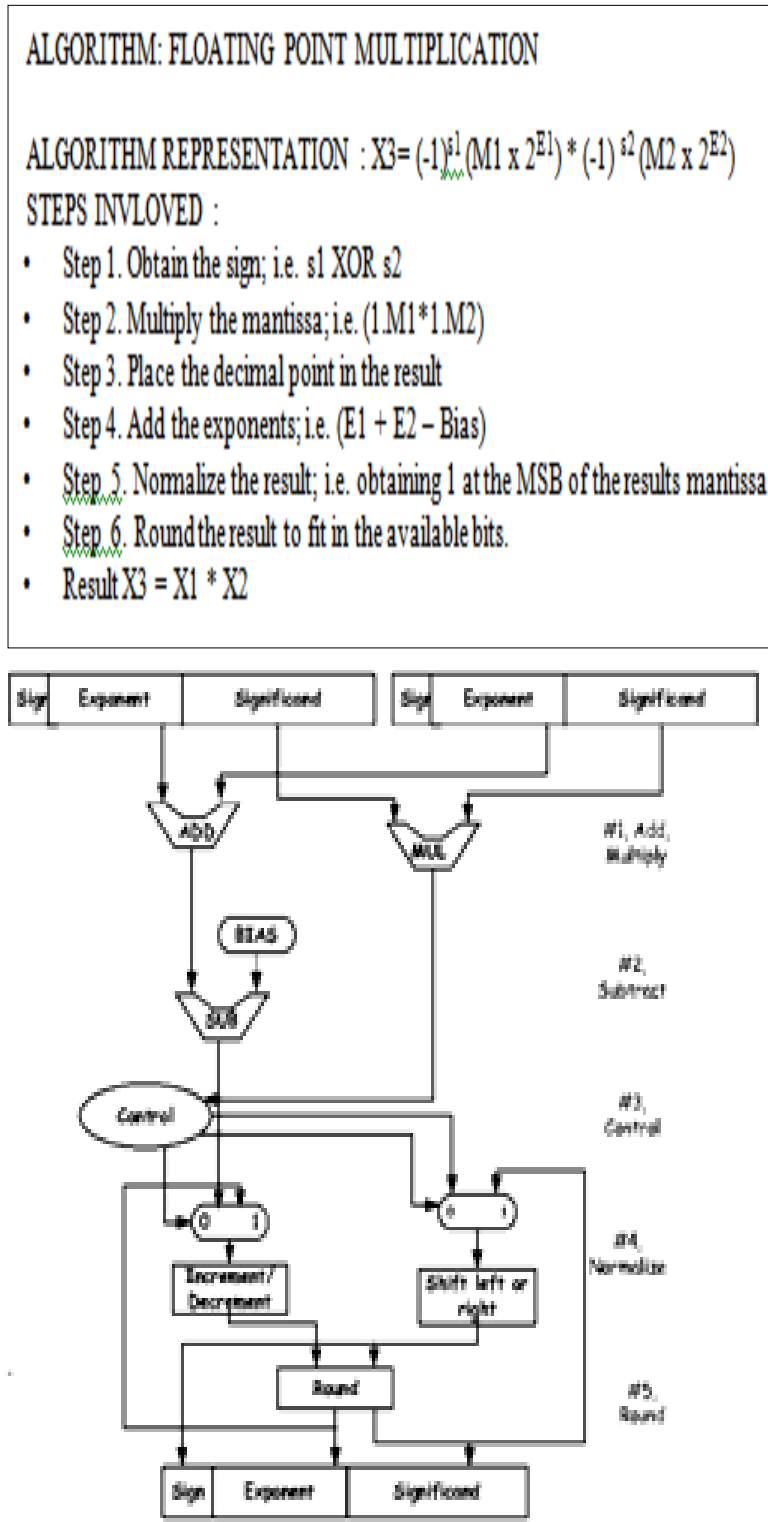

Fig 4 :Floating Point Multiplication Architecture

\section{IMPLEMENTATION OF FLOATING POINT MULTIPLICATION}

The proposed floating point multiplication block is shown figure 5 , the block diagram of multiplier unit comprises of a XOR operation, normalizer block and bias operation. Consider two operands A and B, the XOR involves in SIGN CALCULATION : if its positive " 0 " or " 1 ".

EXPONENT CALCULATION : In this design exponent addition is calculated with two 8 bit exponent and this block is proposed with 8 bit carry look ahead adder (CLA) as shown in figure 6 , it enhances the speed by dropping the time essential to corroborate carry bits. It computes one or added carry bits former to the sum, the huge-value bits of the adder to conclude the outcome has less wait time The end result is a condensed carry propagation time. It subsets of propagate,

sum and carry generator. It adds the exponents of the two operands and then it bias 127 is subtracted from results obtained from the resultant result of exponent i.e.EA+EB-bias .

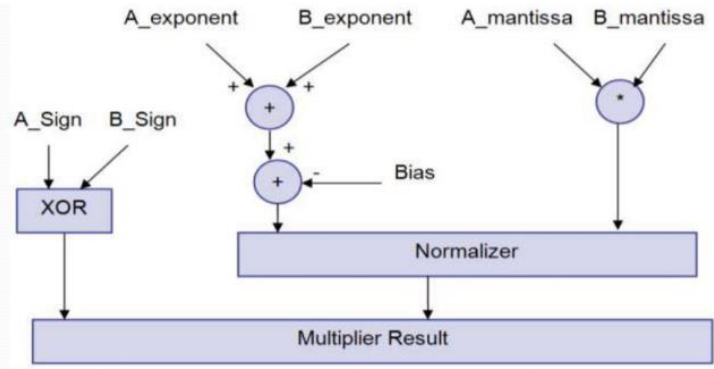

Fig 5: Block Diagram Of Multiplier

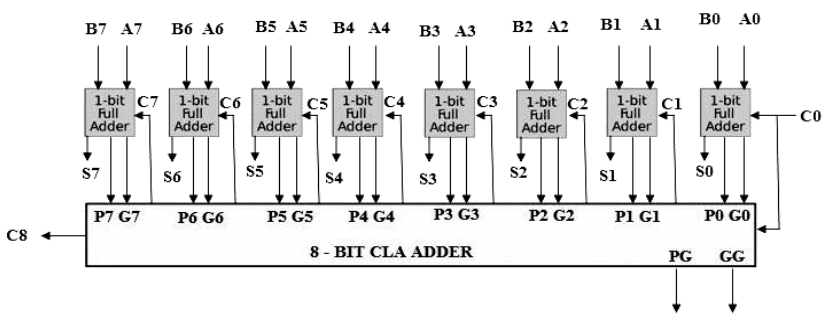

Fig 6 : 8 Bit Carry Look Ahead For Exponent

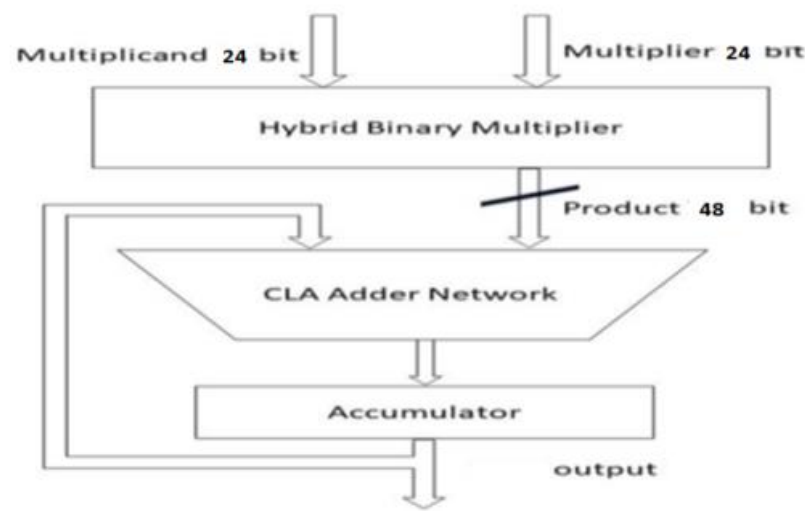

Fig 7 : 24 Bit hybrid multiplier for Mantissa

MANTISSA CALCULATION: In this design the mantissa/significand of two operands $\mathrm{A}$ and $\mathrm{B}$ are to be multiplied. As performance of mantissa multiplier dominates overall performance of the floating point multiplication, proposed 24 bit hybrid multiplier is designed as shown in figure 7.Here ,24 bit operands A and B is given into hybrid multiplier the partial products are given to CLA adder network then those values gets stored in the accumulator then final result of the calculated values is results as the output of 48 bits .Finally, all the resultants of the values of the sign calculation and mantissa calculation and exponent calculation are all formed into a standard IEEE-754 format representation.

\section{FLOATING POINT ADDITION}

Floating point addition (FPA)represented using single precision format in calculating sign bit, exponent and mantissa addition then normalize and round off the resultant obtained to attain the final result. 


\section{Design and Implementation of FPU for Optimised Speed}

Sign bit is calculated by means of XOR operation of two operands sign bit. The exponents of the operands are added to attained though the addition of mantissa .The ensuing sum is subtracted from 127 in single precision to achieved its biased exponent. The architecture of floating point addition 32 bit in figure 8.

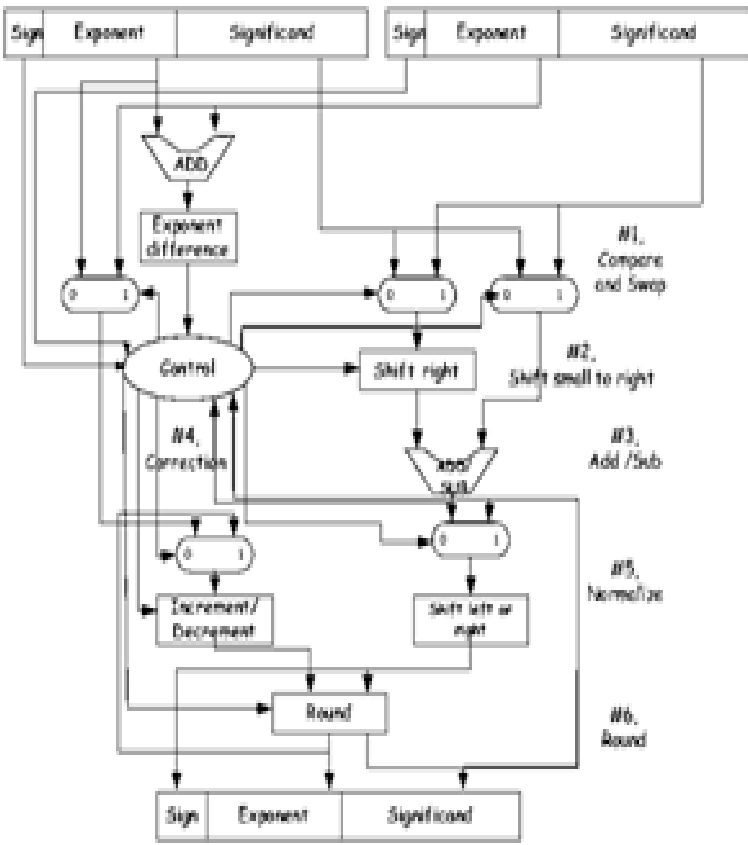

Fig 8 : Floating Point Addition Architecture

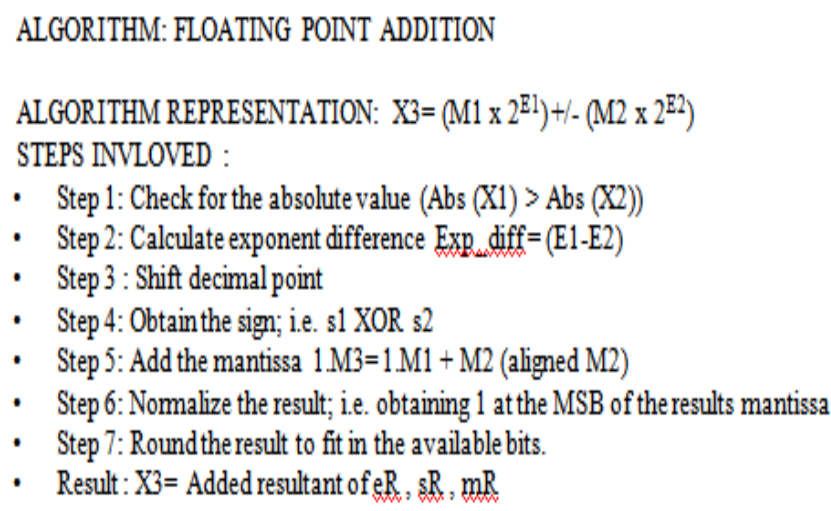

\section{IMPLEMENTATION OF FLOATING POINT ADDITION}

The proposed floating point addition block is shown figure 9, the block diagram of addition unit, this unit comprises of sign calculator, exponent calculator, shifter and rounding and normalizer block. Examine operand A and B,SIGN CALCULATION :If is positive its " 0 " or " 1 " in sign bit . EXPONENT CALCULATION : In this design exponent addition is calculated with two 8 bit exponent and this block is proposed with 8 bit carry look ahead adder (CLA) as shown in figure 10,it boost speed by reducing the time obligatory to confirm carry bits. It reckon one or more carry bits prior to the sum , the result of the substantial-value bits of the adder will achieve less wait time. The outcome is a abridged carry propagation time. It consists of propagate, sum and carry generator. It adds the exponents of the two operands and then it bias 127 is subtracted from results obtained from the resultant result of exponent i.e.EA+EB-bias .

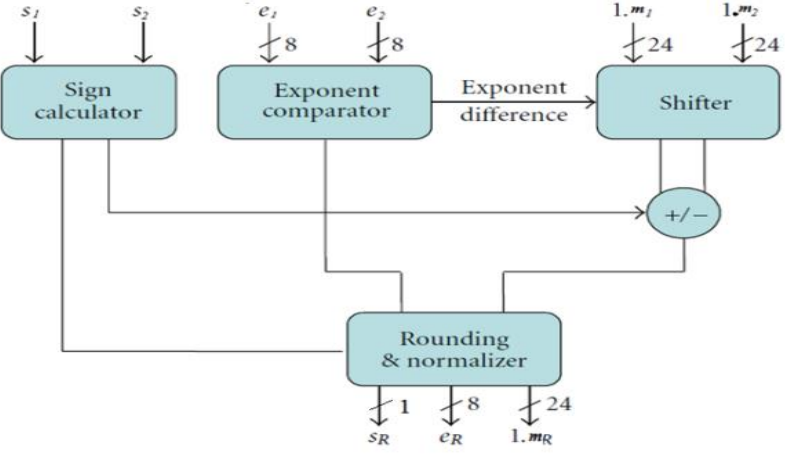

Fig 9 : Block Diagram Of Addition Unit

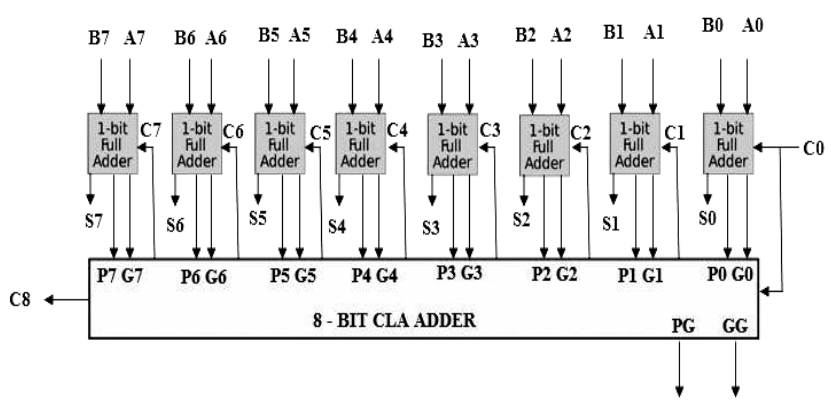

Fig 10 : 8 Bit Carry Look Ahead For Exponent

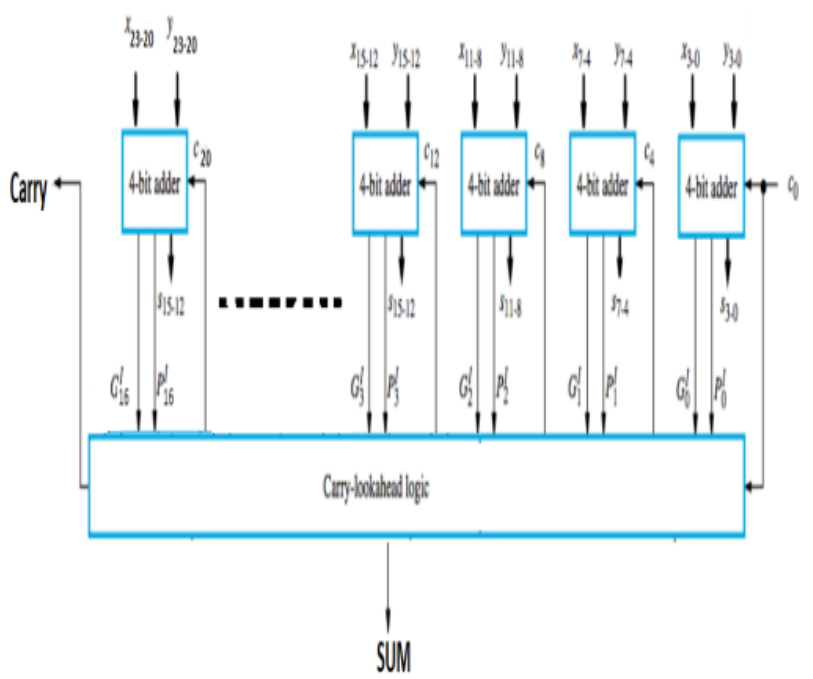

Fig 11 : 24 Bit Carry Look Ahead For Mantissa

MANTISSA CALCULATION: In this design the mantissa/significand of two operands A and B are added. As performance of mantissa adder dominates overall performance of the floating point addition, proposed 24 bit CLA is designed as shown in figure 11.Here ,24 bit operands $A$ and $B$ is given into CLA then final result of the calculated values is results as the output of 48 bits .Finally, all the resultants of the values of the sign calculation and mantissa calculation and exponent calculation are all formed into a standard IEEE-754 format representation.

\section{DOUBLE PRECISION FLOATING POINT}

The IEEE double precision floating point format has a sign bit is represented as " $1 / 0$ " bit. " 1 " in sign bit indicates the negative and " 0 " indicates the positive.

Published By:

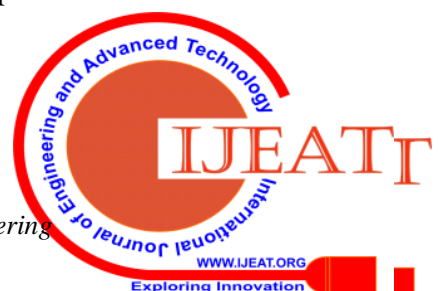


The total number of exponent is 255 here the ranges depends on the exponent value in accordance 11 bits of exponent is accommodated and it has a bias of 1027 .There are totally 53 bits of mantissa but last 1 bit is hidden so it's always represented as 52 bit of mantissa as per IEEE-754 standard and occupies 64 bit.

\section{PIPELINING TECHNIQUE}

Pipelining is one of the mainstream strategies to acknowledge high performance computing stage. It was first introduced by IBM in 1954 in Project Stretch. In a pipelined unit a new task is started before the previous is finished. This is feasible because most of the adders, high speed multipliers are combinational circuits. Formerly a output has been set it remains fixed for the remainder of the operation. Pipelining is a technique to provide additional increase in bandwidth by allowing simultaneous execution of many tasks. To achieve pipelining input processes must be subdivided into a sequence of subtasks, each of which can be executed by dedicated hardware stage that operates along with other stages in the pipeline. The adder design pipelines the steps shifting, addition and normalization to achieve a summing up every clock cycle. Each pipeline stage perform operations autonomous of others. Input data to the added endlessly streams in. Speed of operation of pipelined add has been established 3 times more than the non pipelined addition and multiplication.

\section{IMPLEMENTATION OF FLOATING POINT MULTIPLICATION WITH PIPELINING}

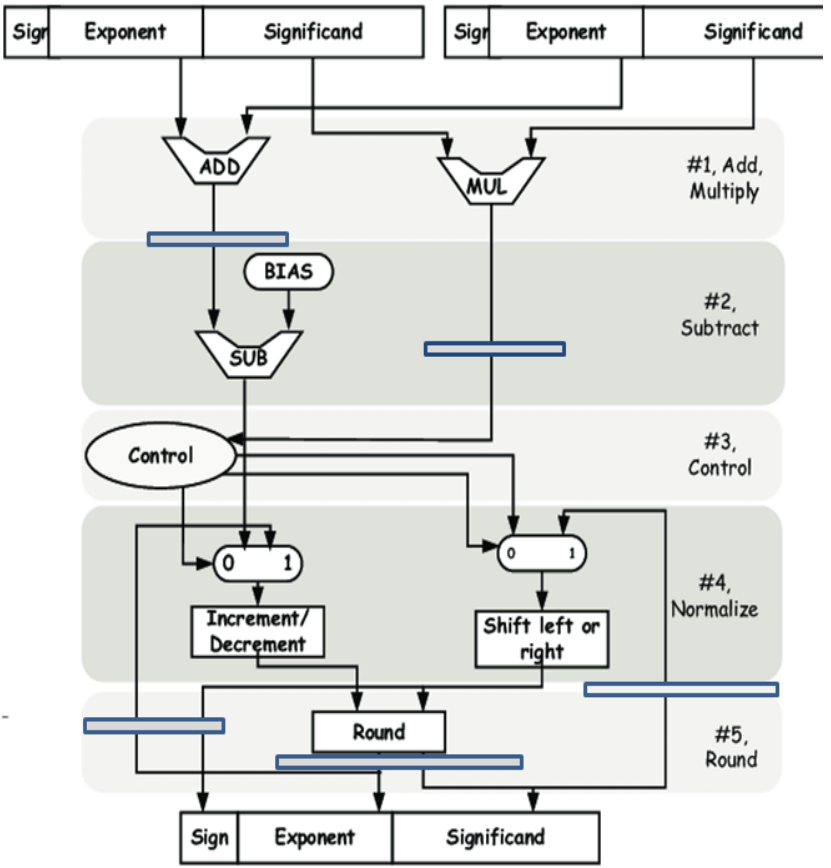

Fig 12 : Pipelined Floating Point Multiplication Architecture

The proposed pipelined floating point multiplication architecture is shown figure 12 .The pipelined register are placed in the task to be performed . Consider two operands A and $\mathrm{B}$, the XOR involves in SIGN CALCULATION : if the number is positive then its " 0 " else " 1 .
EXPONENT CALCULATION : In this design exponent addition is calculated with two 11 bit exponent and the block is proposed with 11 bit carry look ahead adder (CLA) as shown in figure 13 ,it enhance speed by dropping the time requisite to validate carry bits. It calculates one or more carry bits prior to the sum, this reduces the stay time to conclude the result of the larger-value bits of the adder. The consequence is a reduced carry propagation time. It consist of propagate, sum and carry generator. It adds the exponents of the two operands and then it bias (1023) is subtracted from results obtained from the resultant result of exponent i.e.EA+EB-bias .

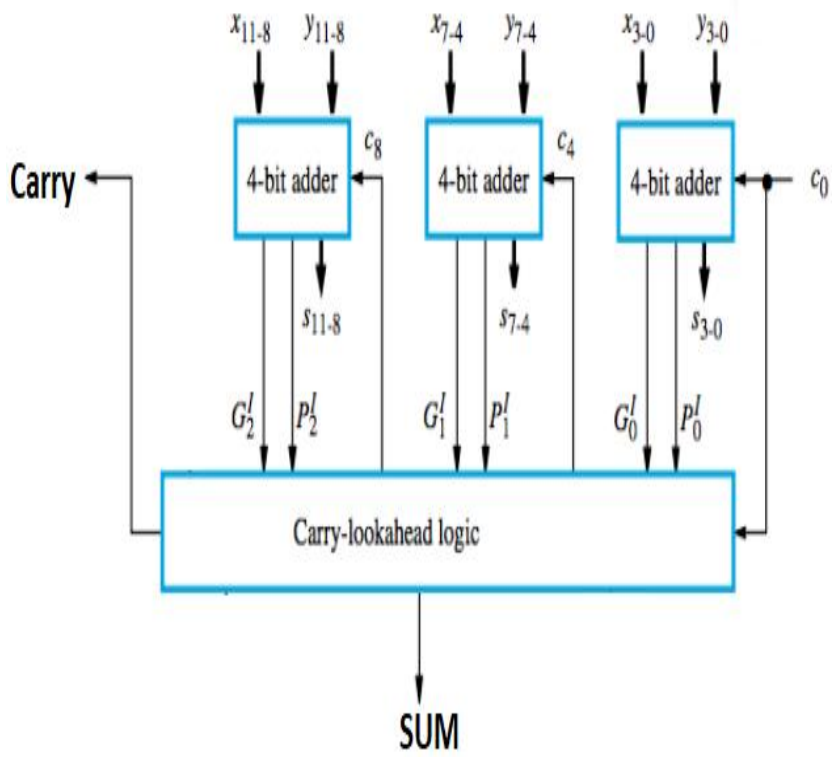

Fig 13 : 11 Bit Carry Look Ahead For Exponent

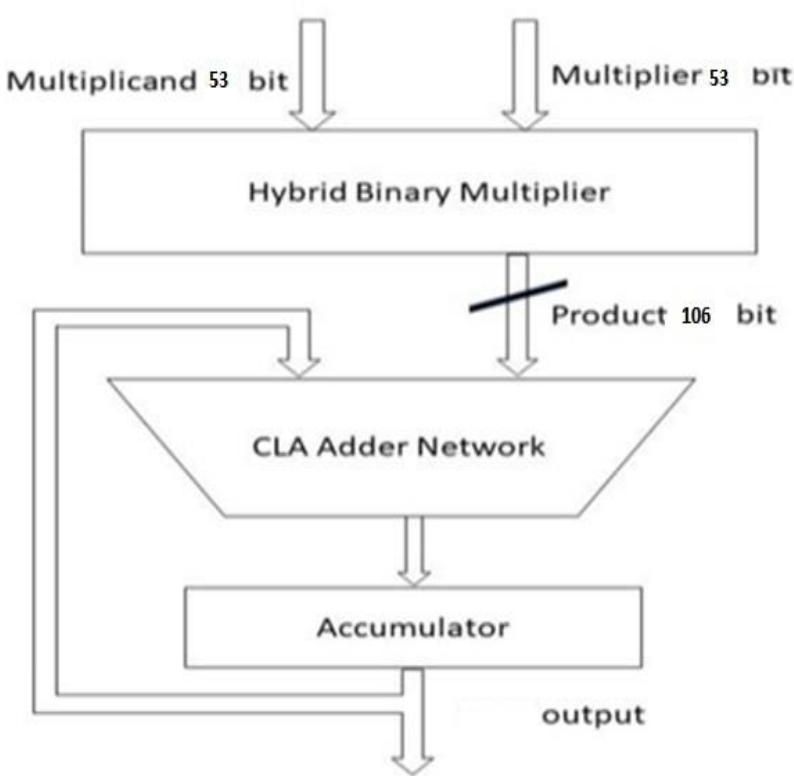

Fig 14: 53 Bit Hybrid Multiplier For Mantissa MANTISSA CALCULATION: In this design operands A and B mantissa/significand are to be multiplied. 
As performance of mantissa multiplier dominates overall performance of the floating point multiplication, proposed 53 bit hybrid multiplier is designed as shown in figure 14.In,53 bit operands A and B is feed into hybrid multiplier the partial products are given to CLA adder network then those values gets stored in the accumulator then final result of the calculated values is results as the output of 106 bits .Finally, all the resultants of the values of the sign calculation and mantissa calculation and exponent calculation are all formed into a standard IEEE-754 format representation.

\section{IMPLEMENTATION OF FLOATING POINT ADDITION WITH PIPELINING}

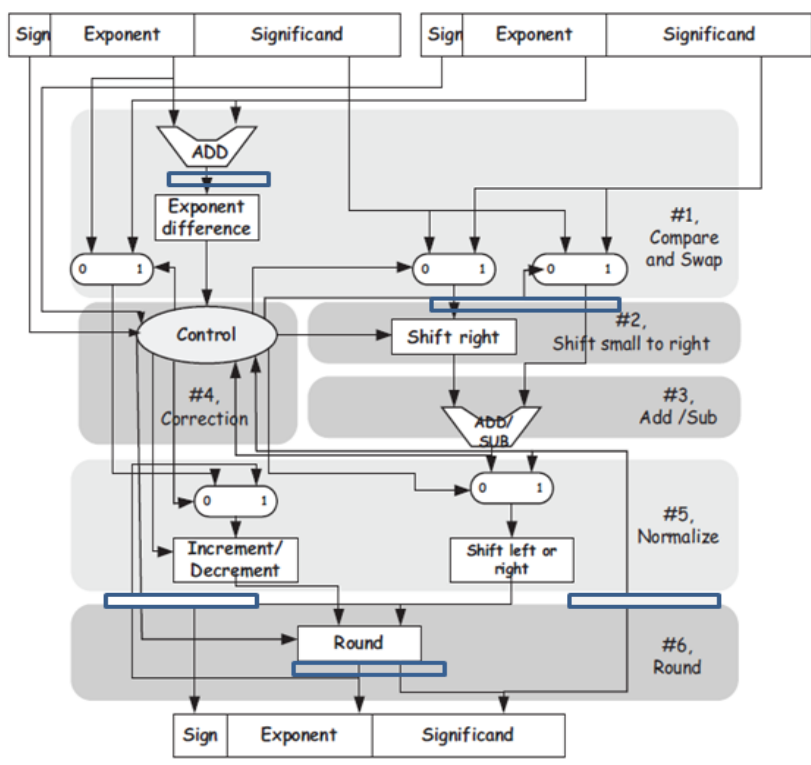

Fig 15 : Pipelined Floating Point Addition Architecture

The proposed pipelined floating point addition architecture is shown figure 15 .The pipelined register are placed in the task to be performed . Consider two operands A and B, the XOR involves in SIGN CALCULATION : if the number is positive then its " 0 " else " 1 .

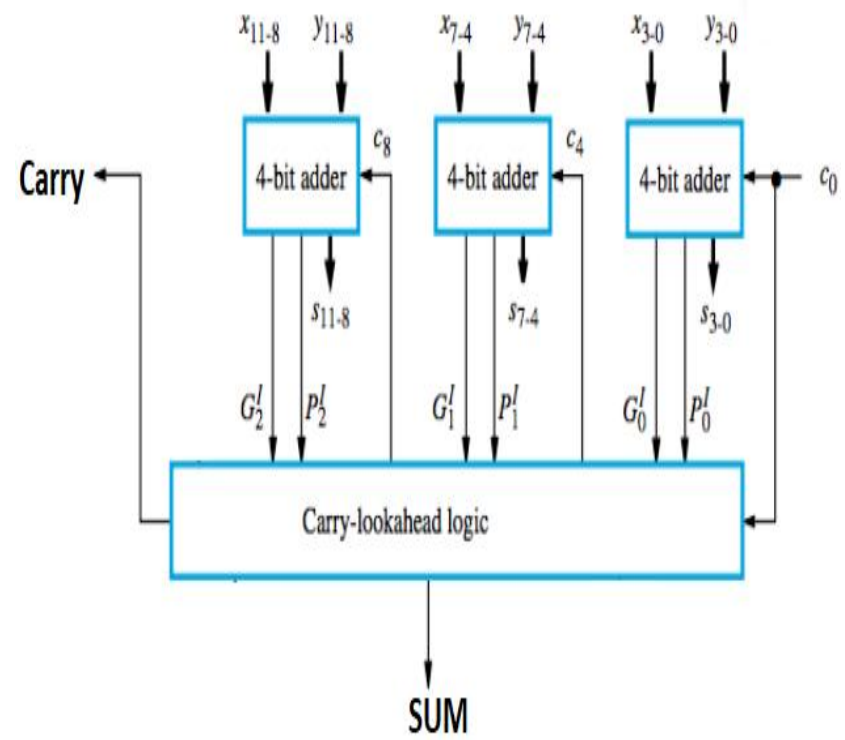

Fig 16 : 11 Bit Carry Look Ahead For Exponent

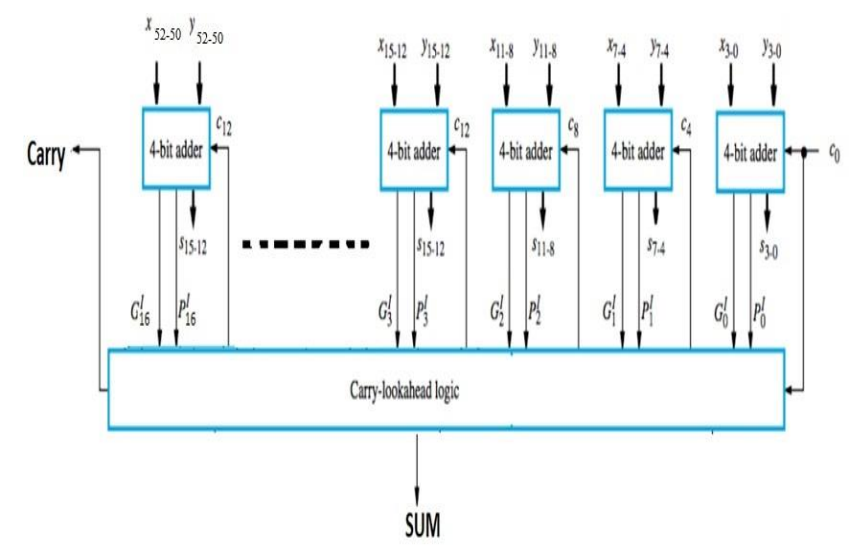

Fig 17 : 53 Bit Carry Look Ahead For Mantissa

EXPONENT CALCULATION : In this design exponent addition is completed with two 11 bit exponent and the block is proposed with 11 bit carry look ahead adder (CLA) as shown in figure 16 , it helps in reducing the sum of time necessary to conclude carry bits. To enumerate one or supplementary carry bits forward of the sum it , the result of the huge-value bits of the adder conclude that its less wait of time. It adds the resultant exponents $\mathrm{A}$ and $\mathrm{B}$ then it subtracted from bias 127 i.e.EA+EB-bias .

MANTISSA CALCULATION: In this design is mantissa / significand of two operands are added. As performance of mantissa adder dominates overall performance of the floating point addition , proposed 53 bit CLA is design is shown in figure 17.The 53 bit operands A and B is feed into CLA then final result of the calculated values is results as the output of 106 bits .Finally ,all the resultants of the values of the sign calculation and mantissa calculation and exponent calculation is followed by a standard IEEE-754 format representation.

\section{SIMULATION RESULTS}

The simulation of proposed single precision and double precision FPM and FPA of internal adder and multiplier has been done to calculate the high throughput. This section includes comparative results of hybrid multiplier and existing multipliers and also existing adders and proposed adder. The input values are converted by a tool [7][8]. Table III shows the comparison of multipliers. Table IV shows the comparison of adders and Table $\mathrm{V}$ shows device summary of proposed single precision FPM.Table VI shows performance analysis of proposed single precision FPM and Table VII shows device summary of proposed single precision FPA.Table VIII shows performance analysis of proposed single precision FPA. Table IX shows device summary of proposed double precision FPM.Table X shows performance analysis of proposed double precision FPM. Table XI shows device summary of proposed double precision FPA.Table XII shows performance analysis of proposed double precision FPA is implemented by using Verilog language and its simulated in Xilinx ISE 14.5i on Spartan 6 . 


\section{SINGLE PRECISION OF FPM}

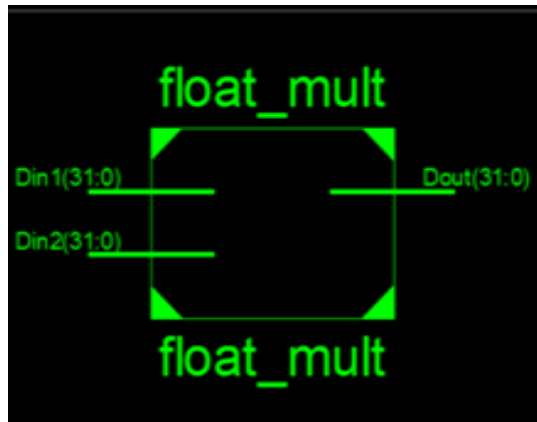

Fig 18 : RTL design of single precision FPM

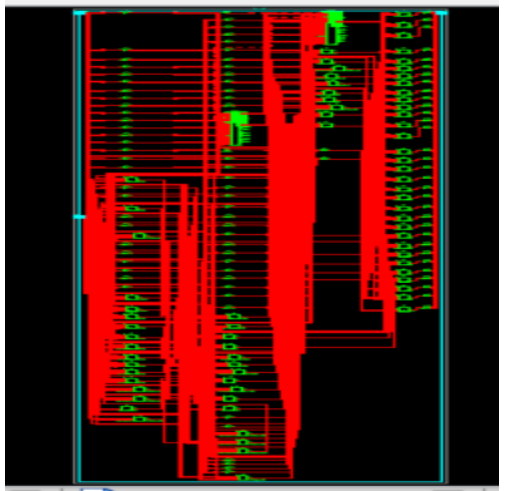

Fig 19: Technology schematic of single precision FPM The proposed single precision FPM is designed. The proposed FPM is integrated internally with the proposed adder and hybrid multiplier and RTL design and technology schematic view is shown in figure18,19. The simulation results of the calculated values is shown in figure 20.

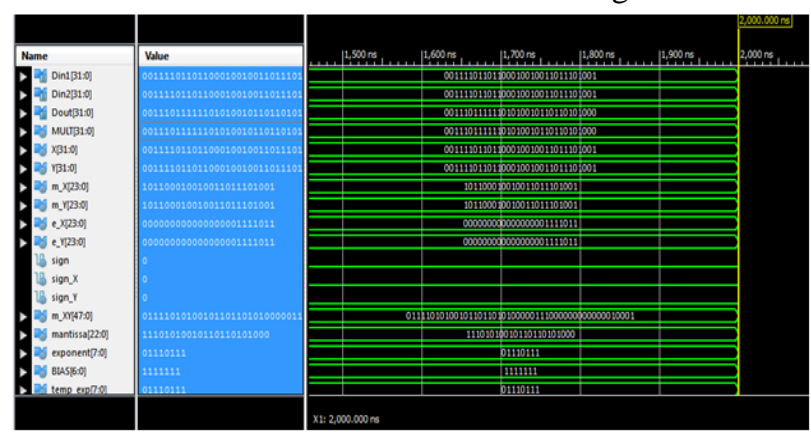

Fig 20 : Simulation results of single precision FPM SINGLE PRECISION FPM: - VALUE APPLIED :0.0865* $0.0865=0.00748225$

Din1-0. $0865=0 \_0111101$ 1_0110001 0010011011101001 Din2- 0.0865 = 0_0111101 1_0110001 0010011011101001 Dout- 0.00748225=0_01110111_ 1110_1010_1001_0110_1101_0100

\section{1(a).INTERNAL ADDER IN FPM FOR EXPONENT}

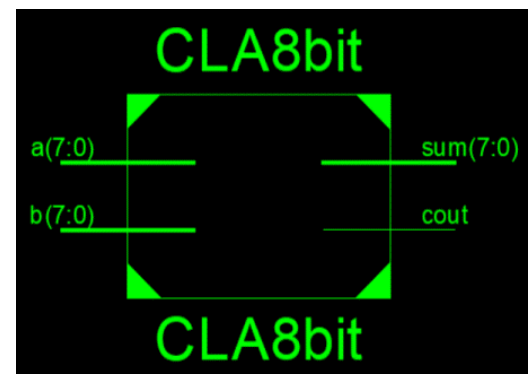

Fig 21 : RTL design 8 bit CLA adder for exponent

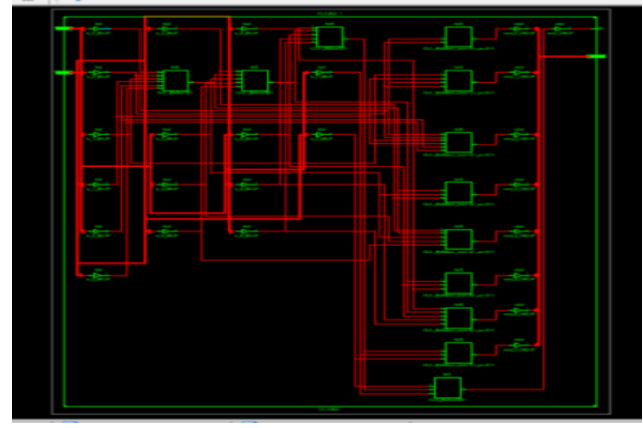

Fig 22 : Technology schematic 8 bit CLA adder for exponent

The proposed single precision FPM is internally designed with CLA adder of 8 bit for exponent part calculation. The RTL design and technology schematic view of the proposed CLA 8 bit is shown in figure 21,22. The simulation results of the calculated values is shown in figure 23 , here value applied in input a is 56 and input b is 34 is added and output sum value is 90 .

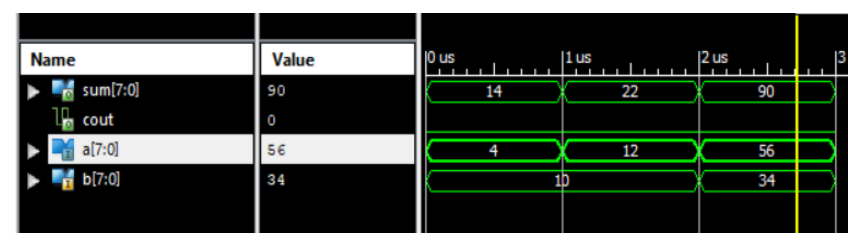

Fig 23: Simulation results of 8 bit CLA adder for exponent

1(b).INTERNAL MULTIPLIER IN FPM IN MANTISSA

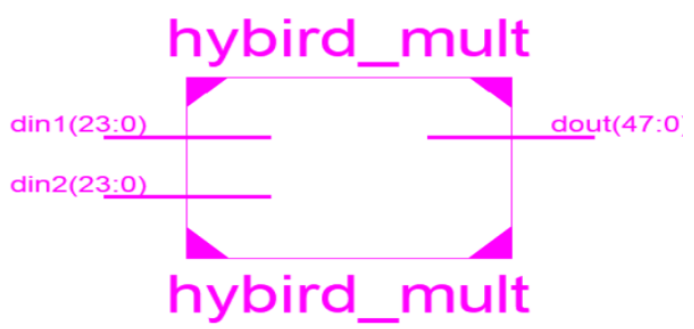

Fig 24 : RTL Design 24 bit Hybrid multiplier for mantissa

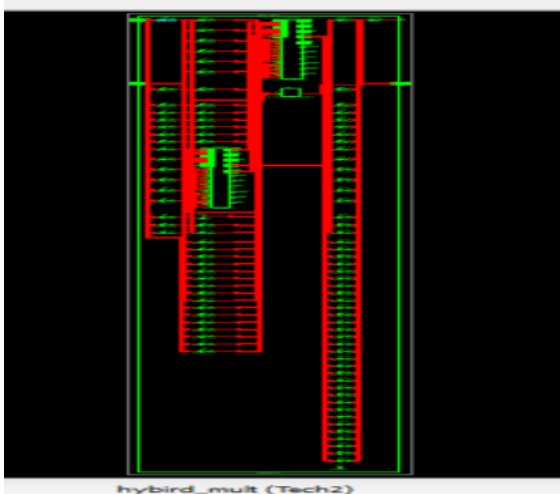

Fig 25: Technology schematic 24 bit Hybrid multiplier for mantissa

The proposed single precision FPM is internally designed with hybrid multiplier of 24 bit for mantissa part calculation .

Published By: 


\section{Design and Implementation of FPU for Optimised Speed}

The RTL design and technology schematic view of the proposed hybrid multiplier of 24 bit is shown in figure 24,25. The simulation results of the calculated values is shown in figure 26, here value applied in input a is 1098 and input b is 1098 is multiplied and the output dout value is 474336 .

\begin{tabular}{|c|c|c|c|c|}
\hline Name & Value & 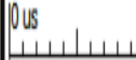 & |lus & |lus \\
\hline D & 1098 & 120 & 453 & 1098 \\
\hline$>$ dinn2[23:0] & 432 & 300 & 560 & 432 \\
\hline 1) dout $44: 0]$ & 474336 & 36000 & 253680 & 474336 \\
\hline
\end{tabular}

Fig 26: Simulation results of 24 bit hybrid multiplier for mantissa

\section{SINGLE PRECISION OF FPA}

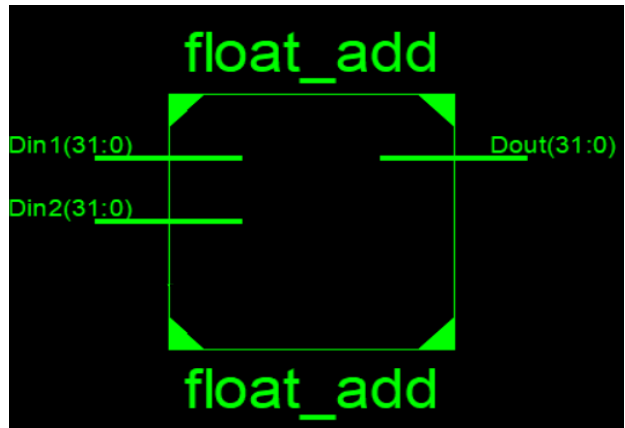

Fig 27 : RTL design of single precision FPA

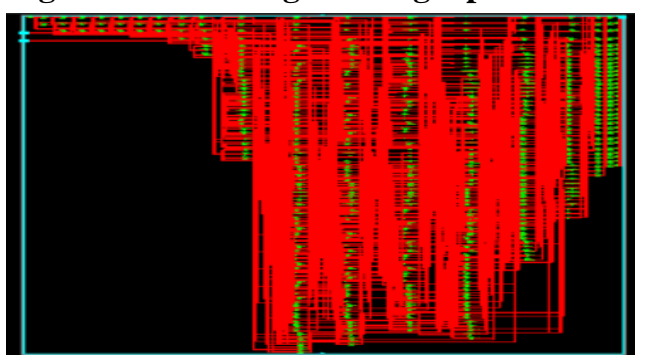

Fig 28 : Technology schematic of single precision FPA The proposed single precision FPA is designed The proposed FPA is integrated internally with the proposed adder and RTL design and technology schematic view is shown in figure 27,28 . The simulation results of the calculated values is shown in figure 29.
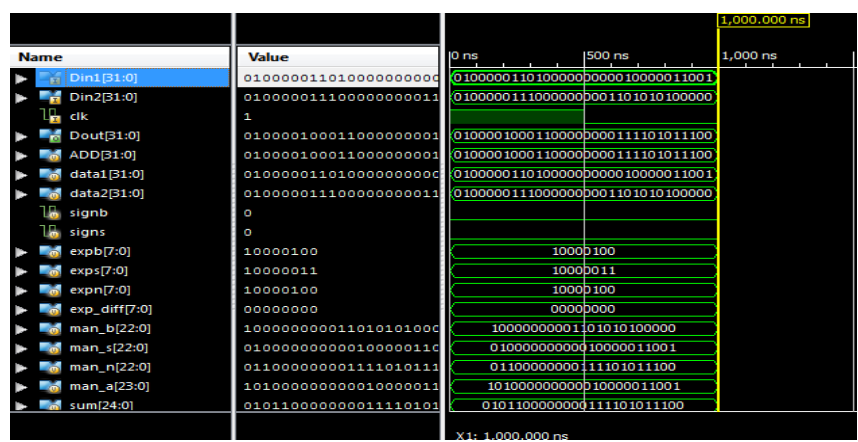

Fig 29 : Simulation results of single precision FPA Single precision FPA:VALUE APPLIED :

$20.002+24.013=44.015$

Din1-20.002 $=010000011010000000000100000110010$ Din2-24.013 $=010000011100000000011010101000000$

Dout-44.015 = 01000010001100000000111101011100
2(a).INTERNAL ADDER IN FPA FOR EXPONENT

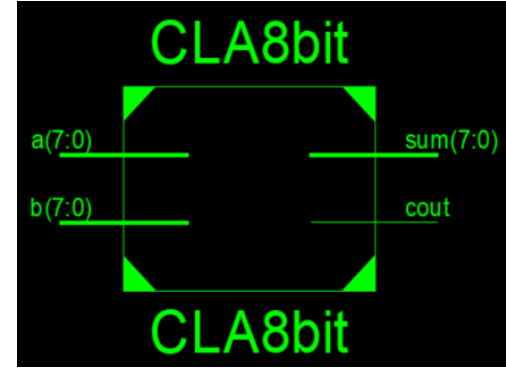

Fig 30 : RTL design 8 bit CLA adder for exponent

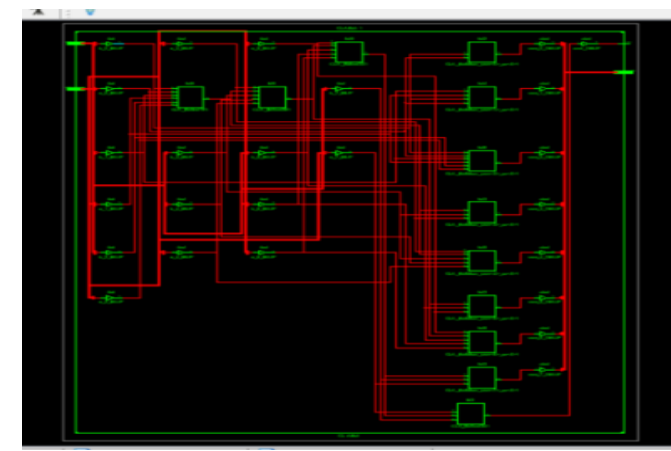

Fig 31 : Technology schematic 8 bit CLA adder for exponent

The proposed single precision FPA is internally designed with CLA adder of 8 bit for exponent part calculation. The RTL design and technology schematic view of the proposed CLA 8 bit is shown in figure 30,31. The simulation results of the calculated values is shown in figure 32 , here value applied in input a is 56 and input b is 34 is added and output sum value is 90 .

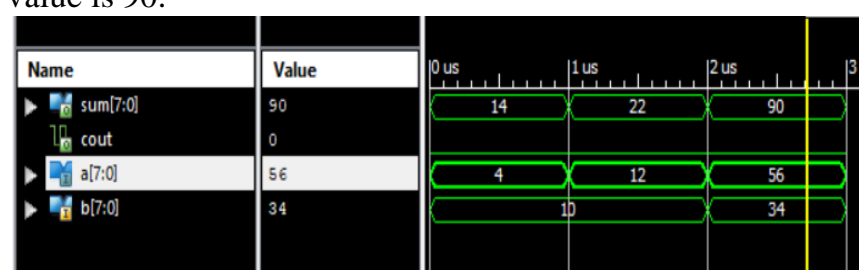

Fig 32: Simulation results of 8 bit CLA adder for exponent

2(b).INTERNAL ADDER IN FPA FOR MANTISSA

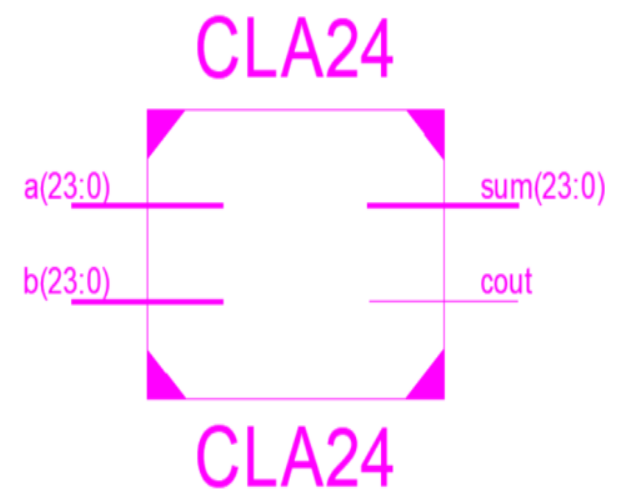

Fig 33 : RTL Design 24 bit CLA for mantissa

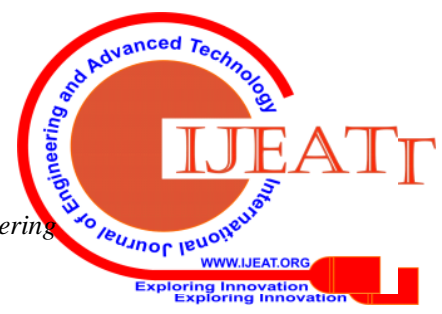




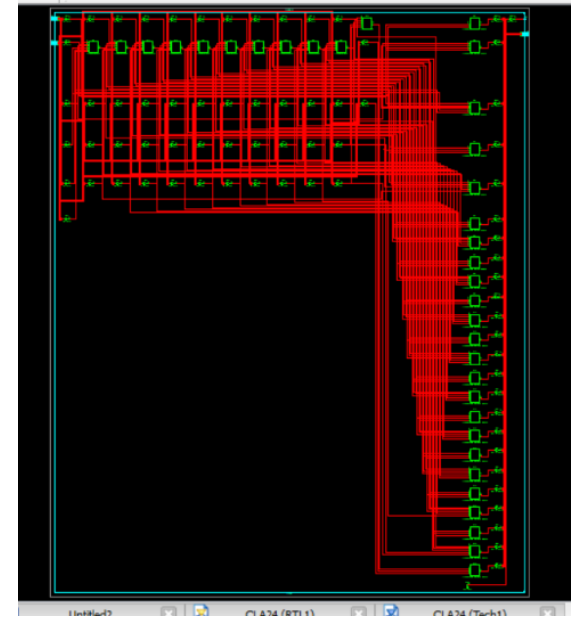

Fig 34: Technology schematic 24 bit CLA for mantissa The proposed single precision FPA is internally designed with CLA 24 bit for mantissa part calculation. The RTL design and technology schematic view of the proposed CLA 24 bit is shown in figure 33,34. The simulation results of the calculated values is shown in figure 35 , here value applied in input a is 125 and input $b$ is 453 is multiplied and the output dout value is 578 .

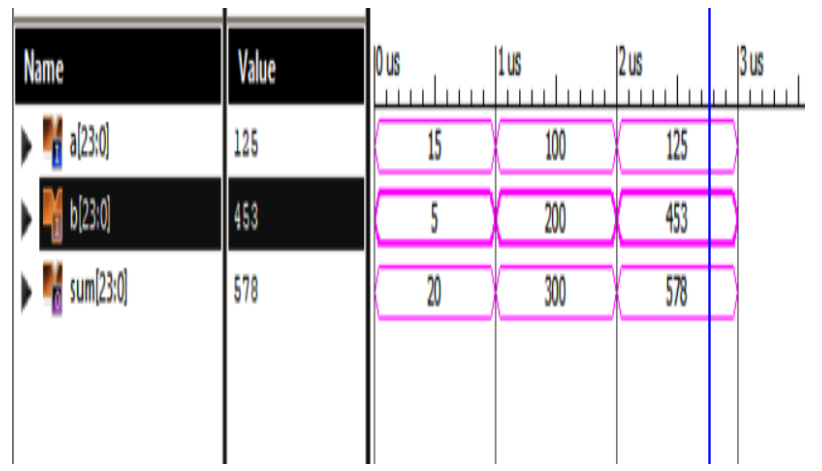

Fig 35: Simulation results of 24 bit CLA for mantissa

\section{DOUBLE PRECISION OF FPM}

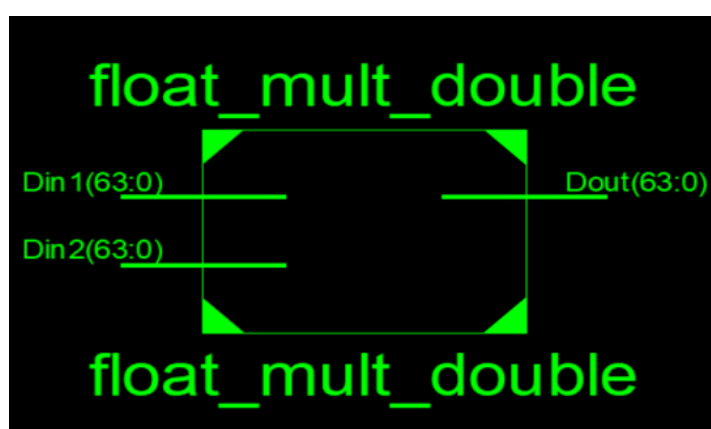

Fig 36 : RTL design of double precision FPM

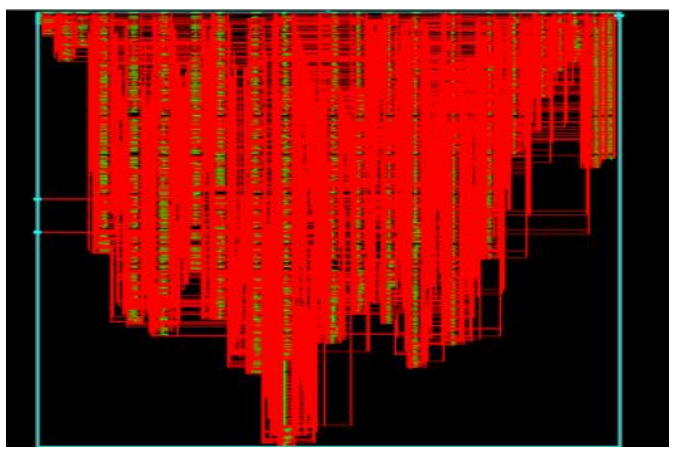

Fig 37: Technology schematic of double precision FPM
The proposed double precision FPM is designed. The proposed FPM is integrated internally with the proposed adder and hybrid multiplier and RTL design and technology schematic view is shown in figure 36,37. The simulation results of the calculated values is shown in figure 38 .

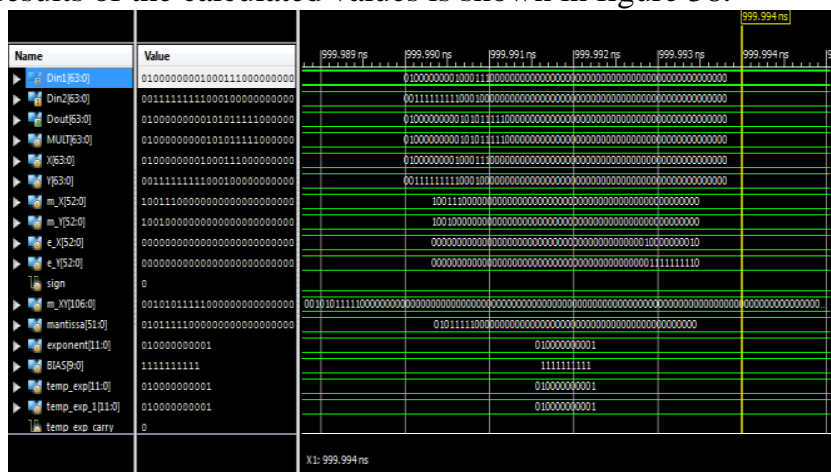

Fig 38 : Simulation results of double precision FPM Double Precision FPM: VALUE APPLIED :0.5625*9.750 $=5.484375$

Din1-0.5625- 0_01111111110_0010_00000000_00000000 _00000000_00000000_00000000_00000000

Din2-9.750- 0_10000000010_0011_10000000_00000000 _00000000_00000000_00000000_00000000

Dout $-5.484375=$

0_10000000001_01011111_0000000000000000000000000 0000000000000000000

3(a). INTERNAL ADDER IN FPM FOR EXPONENT

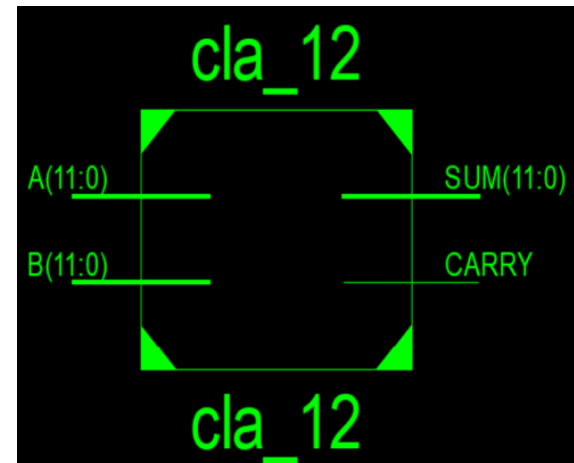

Fig 39 : RTL design 12 bit CLA adder for exponent

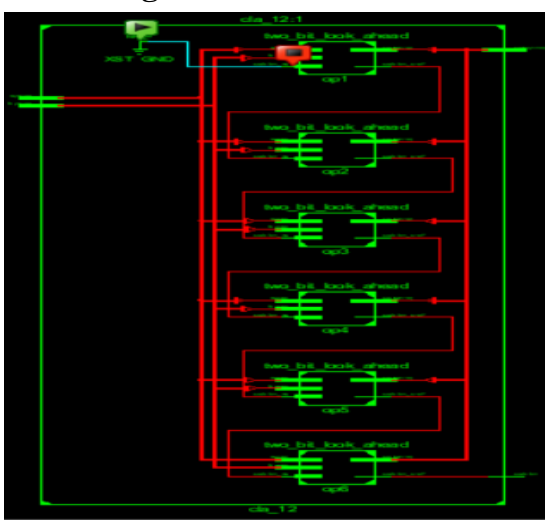

Fig 40: Technology schematic 12 bit CLA adder for exponent

The proposed double precision FPM is internally designed with CLA adder of 12 bit for exponent part calculation. The RTL design and technology schematic view of the proposed CLA 12 bit is shown in figure 39,40 .

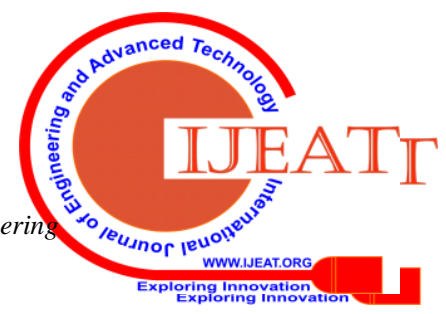




\section{Design and Implementation of FPU for Optimised Speed}

The simulation results of the calculated values is shown in figure 41, here value applied in input a is 26 and input b is 92 is added and output sum value is 118 .

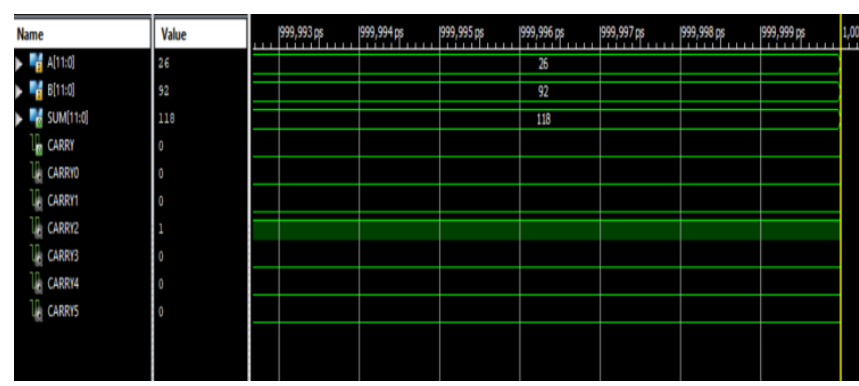

Fig 41: Simulation results of 12 bit CLA adder for exponent

3(b). INTERNAL MULTIPLIER IN FPM FOR MANTISSA

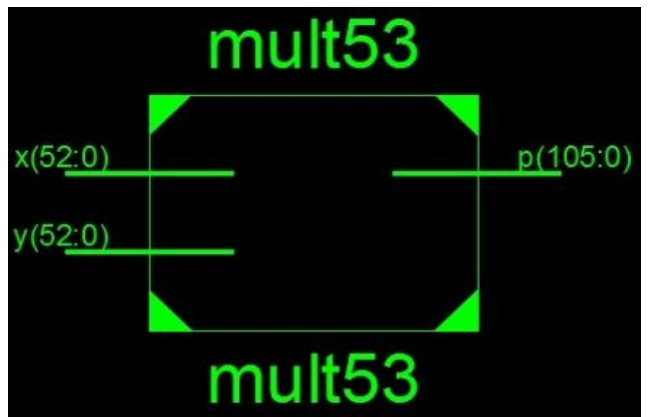

Fig 42: RTL Design 53 bit Hybrid multiplier for mantissa

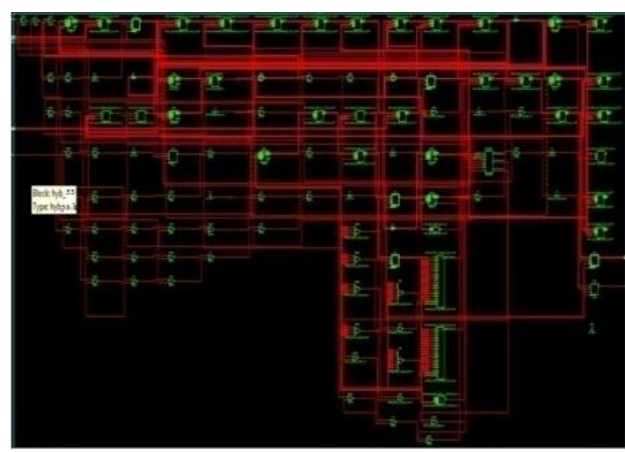

Fig 43: Technology schematic of 53 bit Hybrid multiplier for mantissa

The proposed double precision FPM is internally designed with hybrid multiplier of 53 bit for mantissa part calculation The RTL design and technology schematic view of the proposed hybrid multiplier of 53 bit is shown in figure 42,43. The simulation results of the calculated values is shown in figure 44, here value applied in input a is 33 and input b is 10 is multiplied and the output dout value is 330 .
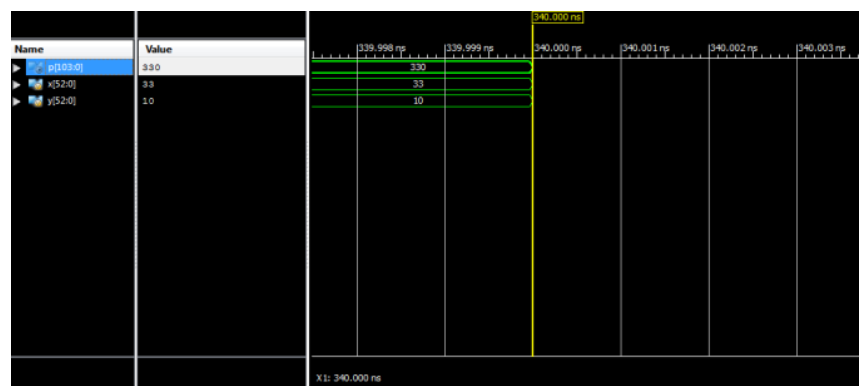

Fig 44: Simulation results of 53 bit Hybrid multiplier for mantissa

\section{DOUBLE PRECISION OF FPA}

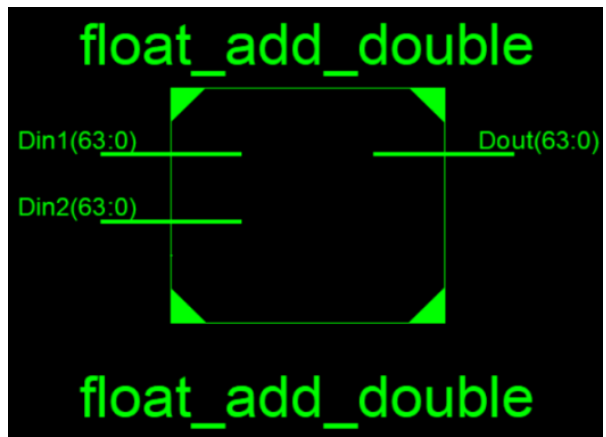

Fig 45: RTL design of double precision FPA

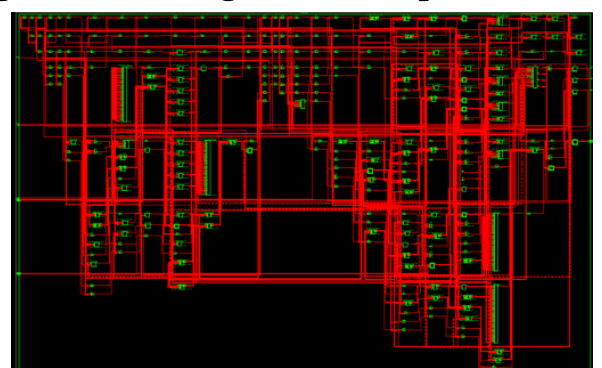

Fig 46: Technology schematic of double precision FPA . The proposed double precision FPA is designed. The proposed FPA is integrated internally with the proposed adder and RTL design and technology schematic view is shown in figure 45,46. The simulation results of the calculated values is shown in figure 47

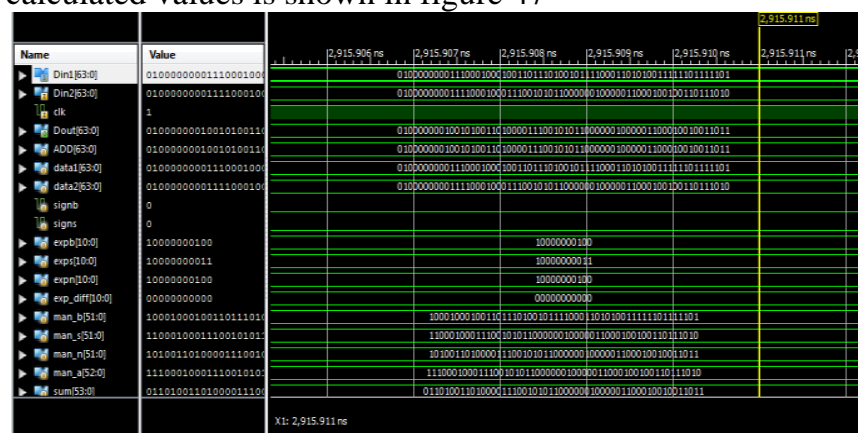

Fig 47: Simulation results of double precision FPA Double Precision FPA: VALUE APPLIED : $24.538+28.278=52.816$

Din1-24.538=0100000001001000 1000100110111010 01011110001101010011111101111101

Din2-28.278=-0100000000111100 0100011100101011 00000010000011000100100110111010

Dout $-52.816=0100000001001010011010000111001010110$ 000001000001100010010011100

\section{4(a).INTERNAL ADDER IN FPA FOR EXPONENT}

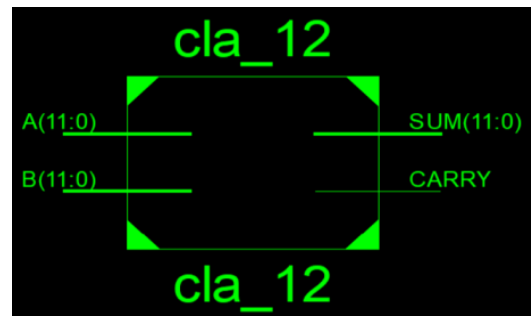

Fig 48 : RTL design 12 bit CLA adder for exponent

Published By:
Blue Eyes Intelligence Engineering \& Sciences Publication 
The proposed double precision FPA is internally designed with CLA adder of 12 bit for exponent part calculation. The RTL design and technology schematic view of the proposed CLA 12 bit is shown in figure 48,49. The simulation results of the calculated values is shown in figure 50 , here value applied in input a is 26 and input b is 92 is added and output sum value is 118 .

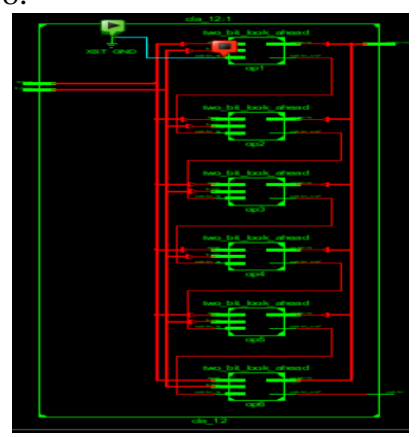

Fig 49: Technology schematic 12 bit CLA adder for exponent

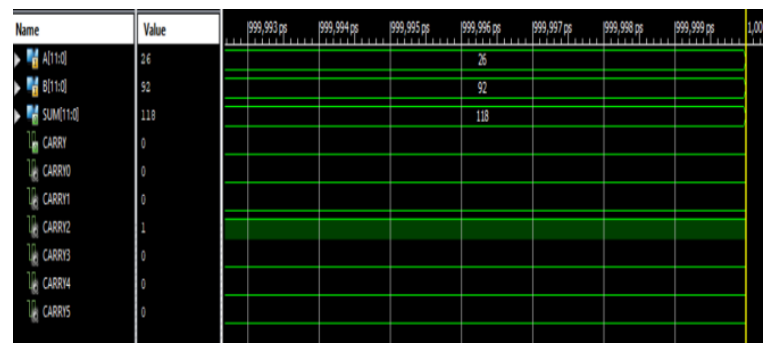

Fig 50: Simulation results of 12 bit CLA adder for exponent

4(b).INTERNAL ADDER IN FPA FOR MANTISSA

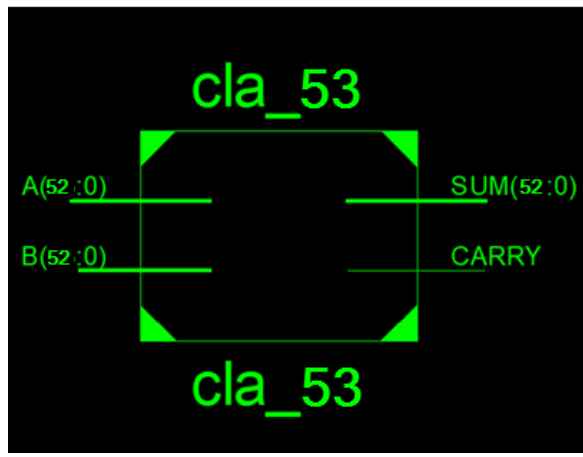

Fig 51 : RTL Design 53 bit CLA for mantissa

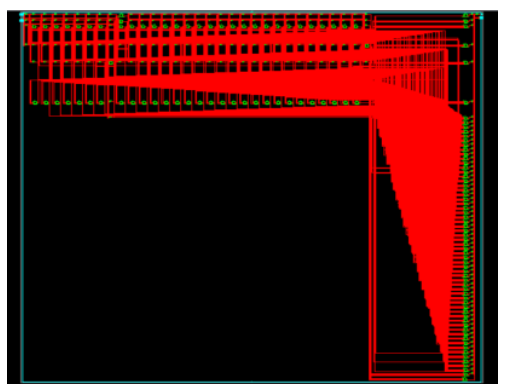

Fig 52: Technology schematic 53 bit CLA for mantissa

The proposed double precision FPA is internally designed with CLA 53 bit for mantissa part calculation. The RTL design and technology schematic view of the proposed CLA 53 bit is shown in figure 51,52. The simulation results of the calculated values is shown in figure 53 , here value applied in input a is 500 and input $b$ is 250 is added and the output dout value is 750 .

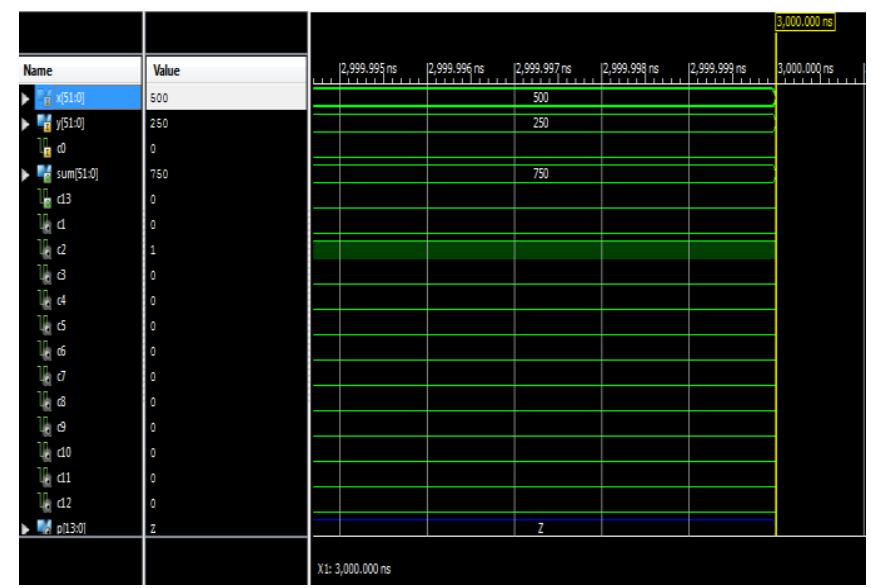

Fig 53: Simulation results of 53 bit CLA for mantissa

TABLE III : Comparison of multipliers

\begin{tabular}{|c|c|}
\hline MULTIPLIERS & DELAY \\
\hline ARRAY MULTIPLIER & $12.150 \mathrm{~ns}$ \\
\hline WALLACE MULTIPLIER & $7.815 \mathrm{~ns}$ \\
\hline BOOTHS & $7.583 \mathrm{~ns}$ \\
\hline $\begin{array}{c}\text { PROPOSED - HYBRID } \\
\text { MULTIPLIER }\end{array}$ & $7.045 \mathrm{~ns}$ \\
\hline
\end{tabular}

TABLE IV: Comparison of adders

\begin{tabular}{|c|c|}
\hline ADDERS & DELAY \\
\hline $\begin{array}{c}\text { CARRY SELECT } \\
\text { ADDER(CSLA) }\end{array}$ & $3.504 \mathrm{~ns}$ \\
\hline CARRY SKIP ADDER(CSA) & $2.925 \mathrm{~ns}$ \\
\hline PROPOSED - CARRY & $2.527 \mathrm{~ns}$ \\
LOOK AHEAD ADDER(CLA) & \\
\hline
\end{tabular}

TABLE V: Device summary of proposed single precision FPM

\begin{tabular}{|l|c|c|c|c|}
\hline PRECSION & $\begin{array}{c}\text { TARGET } \\
\text { FPGA }\end{array}$ & $\begin{array}{c}\text { NO OF } \\
\text { SLICE } \\
\text { FLIP } \\
\text { FLOPS }\end{array}$ & $\begin{array}{c}\text { NO OF } \\
\text { SLICES }\end{array}$ & $\begin{array}{c}\text { NO OF } \\
\mathbf{4} \\
\text { INPUT } \\
\text { LUTS }\end{array}$ \\
\hline $\begin{array}{l}\text { SINGLE } \\
\text { PRECISION }\end{array}$ & SPARTAN 6 & 190 & 308 & 600 \\
\hline
\end{tabular}

TABLE VI: Performance analysis of proposed single precision FPM

\begin{tabular}{|c|c|c|c|}
\hline PRECISION & $\begin{array}{c}\text { TARGET } \\
\text { FPGA }\end{array}$ & FREQUENCY & DELAY \\
\hline $\begin{array}{c}\text { SINGLE } \\
\text { PRECISION }\end{array}$ & SPARTAN 6 & $70.8 \mathrm{MHz}$ & $15.6 \mathrm{~ns}$ \\
\hline
\end{tabular}

TABLE VII: Device summary of proposed double precision FPM

\begin{tabular}{|l|c|c|c|c|}
\hline PRECSION & TARGET & NO OF & NO OF & NO OF \\
& FPGA & $\begin{array}{c}\text { SLICE } \\
\text { FLIP } \\
\text { SLICES }\end{array}$ & $\begin{array}{c}\mathbf{4} \\
\text { INPUT } \\
\text { LUTS }\end{array}$ \\
\hline $\begin{array}{l}\text { DOUBLE } \\
\text { PRECISION }\end{array}$ & SPARTAN 6 & 200 & 400 & 740 \\
\hline
\end{tabular}




\section{Design and Implementation of FPU for Optimised Speed}

TABLE VIII: Performance analysis of proposed double precision FPM

\begin{tabular}{|c|c|c|c|}
\hline PRECISION & $\begin{array}{c}\text { TARGET } \\
\text { FPGA }\end{array}$ & FREQUENCY & DELAY \\
\hline DOUBLE & SPARTAN 6 & $82.5 \mathrm{MHz}$ & $16.12 \mathrm{~ns}$ \\
PRECISION & & & \\
\hline
\end{tabular}

TABLE IX: Device summary of proposed single precision FPA

\begin{tabular}{|l|c|c|c|c|}
\hline PRECSION & $\begin{array}{c}\text { TARGET } \\
\text { FPGA }\end{array}$ & $\begin{array}{c}\text { NO OF } \\
\text { SLICE } \\
\text { FLIP } \\
\text { FLOPS }\end{array}$ & $\begin{array}{c}\text { NO OF } \\
\text { SLICES }\end{array}$ & $\begin{array}{c}\text { NO OF 4 } \\
\text { INPUT } \\
\text { LUTS }\end{array}$ \\
\hline $\begin{array}{l}\text { SINGLE } \\
\text { PRECISION }\end{array}$ & $\begin{array}{c}\text { SPARTAN } \\
6\end{array}$ & 90 & 308 & 600 \\
\hline
\end{tabular}

TABLE X: Performance analysis of proposed single precision FPA

\begin{tabular}{|c|c|c|c|}
\hline PRECISION & $\begin{array}{c}\text { TARGET } \\
\text { FPGA }\end{array}$ & FREQUENCY & DELAY \\
\hline SINGLE & SPARTAN 6 & $75.8 \mathrm{MHz}$ & $15.3 \mathrm{~ns}$ \\
PRECISION & & & \\
\hline
\end{tabular}

TABLE XI: Device summary of proposed double precision FPA

\begin{tabular}{|c|c|c|c|c|}
\hline PRECSION & TARGET & NO OF & NO OF & NO OF 4 \\
FPGA & $\begin{array}{c}\text { SLICE } \\
\text { FLIP } \\
\text { FLOPS }\end{array}$ & $\begin{array}{c}\text { SLICES } \\
\text { LUTS }\end{array}$ \\
\hline DOUBLE & SPARTAN 6 & 100 & 320 & 640 \\
PRECISION & & & \\
\hline
\end{tabular}

TABLE XII: Performance analysis of proposed double precision FPA

\begin{tabular}{|c|c|c|c|}
\hline PRECISION & $\begin{array}{c}\text { TARGET } \\
\text { FPGA }\end{array}$ & FREQUENCY & DELAY \\
\hline DOUBLE & SPARTAN 6 & $86.5 \mathrm{MHz}$ & $20.10 \mathrm{~ns}$ \\
PRECISION & & & \\
\hline
\end{tabular}

\section{XIV.CONCLUSION}

The demand for high performance and throughput and less delay Floating Point Multiplier (FPM) and Floating Point addition (FPA) unit has been on the rise during the recent years. So the study and assessment of different adders and multipliers were considered. To overcome the challenges such as more delay and less throughput, the proposed with hybrid multiplier and adder are designed and integrated into internal modules of Floating Point multiplier(FPM) and Floating Point Addition (FPA) of FPU of single and double precision formats.

\section{FUTURE SCOPE}

The proposed FPM and FPA module of FPU is designed for single precision floating point numbers.It can be further extended for 128 bit and can also be validated in additional modules respectively. Even it can be designed and tried in hardware for even better analysis. In fact by combining hybrid multipliers and adders and additional blocks that can achieve less delay and high throughput.

\section{REFERENCES}

1. D.Goldberg, "What every computer scientist should know about floating-point arithmetic",ACM Computing Surveys Vol.23-1,pp.5-48,1991.
2. L.Louca,T.A.Cook and W.H.Johnson,'Implementation of IEEE Single Precision Floating point Addition and Multiplication On FPFAs",Proceedings of $83^{\text {rd }}$ IEEE 754 Symposium on FPGAs for Custom Computing Machines (FCCM"96),(1996),pp.107-116.

3. B.Parhami,"Computer Arithmetic Algorithm :Algorithms and Hardware Designs", Oxford University Press, 2000.

4. T.Menakadevi, M.Madheswaran," Direct Digital Synthesizer using Pipelined CORDIC Algorithm for Software Defined Radio", International Journal of Science and Technology, Volume 2 No.6, June 2012,pp.372-378.

5. Prerna ,"VHDL Implementation of Addition and Subtraction unit for Floating Point Arithmetic Unit “, International Journal for Research in Technological Studies (IJRTS) on 2014,pp.31-34.

6. https://www.techopedia.com/definition/13051/pipelining

7. Conversion

Toolhttps://babbage.cs.qc.cuny.edu/IEEE-754.old/64bit.html

8. Conversion Tool http://www.binaryconvert.com/convert float.html

9. Rashmi Rahul Kulkarni," Comparison among Different Adders", IOSR Journal of VLSI and Signal Processing (IOSR-JVSP) Volume 5 , Issue 6, Ver. I (Nov -Dec. 2015), PP 01-06

10. https://www.johndcook.com/blog/2009/04/06/anatomy-of-a-floatingpoint-number/

11. http://www.applied-mathematics.net/miniSSEL1BLAS/float-ieee754. pdf

\section{AUTHORS PROFILE}

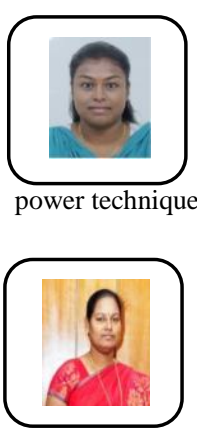

R. Bhuvanapriya, received her B Tech (Electronics and Communication Engineering) degree from Bharath University ,India , in 2017.She is pursuing ME VLSI DESIGN in electronics from Adhiyamaan college of engineering, India.Her research interest include low ,digital signal processing and VLSI .

Menakadevi T., received her BE (Electronics and Communication Engineering) degree from Madurai Kamaraj University and M. Tech (VLSI Systems) degree from NIT, Tiruchirappalli. She obtained her PhD degree in Software Defined Radio Technology from Anna University, India. She is currently working as a Professor in the Department of Electronics and Communication Engineering, Adhiyamaan College of Engineering Hosur, India. Her research interest is VLSI System, SDR, Wireless Communication, Embedded Systems and MANET. She has published more than 70 research papers in referred National and International Journals. 